\title{
Effects of Early Physical and Pulmonary Rehabilitation for Severely and Critically ill COVID-19 Patients: A Retrospective, Cohort, and Multicenter Study
}

Di Qi

Second Affiliated Hospital of Chongqing Medical University

Xiaofeng Yan

Chongqing Public Health Medical Center

Jianglin Xiang

Chongqing Three Gorges Central Hospital

Junnan Peng

Second Affiliated Hospital of Chongqing Medical University

Qian Yu

Second Affiliated Hospital of Chongqing Medical University

\section{Xumao Tang}

Second Affiliated Hospital of Chongqing Medical University

Jin Tong

Second Affiliated Hospital of Chongqing Medical University

Longhua Feng

Qianjiang Central Hospital of Chongqing

Jing Yuan

Chongqing Public Health Medical Center

Yuyan Song

Chongqing Public Health Medical Center

\section{Guodan Yuan}

Chongqing Public Health Medical Center

Daoxin Wang ( $\square$ wangdaoxin0163@163.com )

Second affiliated hospital of Chongqing medical university

\section{Research}

Keywords: Coronavirus Infection, SARS-CoV-2, COVID-19, Rehabilitation, Critical Illness

Posted Date: September 8th, 2020

DOI: https://doi.org/10.21203/rs.3.rs-66798/v1

License: (9) (1) This work is licensed under a Creative Commons Attribution 4.0 International License. Read Full License 


\section{Abstract}

\section{Background}

Effects of early rehabilitation therapy in severe and critical COVID-19 patients remains to be elucidated.

\section{Methods}

We recruited 80 severely and critically ill COVID-19 patients in Chongqing from January 21 to March 15, 2020, who had received rehabilitation therapies or standard treatments within $72 \mathrm{~h}$ of intensive care unit (ICU) admission. We analyzed mortality rates, length of stay in the ICU and hospital, ventilator-free days, and adverse events during hospitalization. Respiratory function, independent functional status, muscle strength, exercise capacity and life quality were measured at hospital discharge and during a three-month follow-up.

\section{Results}

We found no significant differences between the intervention and control groups in terms of ICU, hospital, and 28-day mortality rates, and lengths of stay in the ICU and hospital. Additionally, early rehabilitation enhanced the duration of ventilator-free days, with no increased adverse events and complications. Total lung capacity and carbon monoxide diffusion capacity, Barthel index, and functional independence measure for patients in the intervention group were all higher than those in the control group at hospital discharge and one month after discharge. Compared to the control group, patients in the intervention group had significantly higher Medical Research Council scores and greater walking distance capacities within $6 \mathrm{~min}$ at hospital discharge, as well as one month and three months after discharge. The St. George's Respiratory Questionnaire score was lower in the intervention group than in the control group at one month and three months after hospital discharge.

\section{Conclusions}

Early physical and pulmonary rehabilitation is safe and effective for severely and critically ill COVID-19 patients to promote their functional, physical, and psychological recovery.

\section{Background}

Presently, the global spread of COVID-19 has caused over 700,000 deaths. Coronavirus infections can cause illnesses ranging from a mild cold to severe respiratory infections, such as severe acute respiratory syndrome (SARS). Previous evidence showed that many patients who recovered from SARS complained of limitations in physical function from general weakness to dyspnea in the early rehabilitation phase, which is consistent with lung function abnormalities ${ }^{1}$. COVID-19 is a highly infectious disease that leads to respiratory, physical, and psychological dysfunctions, especially for severely ill patients. It is reported that $5 \%$ of COVID-19 patients are severe patients who require intensive care unit (ICU) care, and $71 \%$ are critically ill patients with hypoxemic respiratory failure, acute respiratory distress syndrome (ARDS) or sepsis requiring treatment with mechanical ventilation ${ }^{2,3}$.On that note, ARDS survivors continue to have functional disabilities one year after discharge from the ICU ${ }^{4,5}$. Additionally, most ARDS patients have extrapulmonary conditions, with muscle wasting and weakness being the most prominent issues ${ }^{6}$. Impaired muscle function and loss of muscle mass, due to routinely immobilization and managed sedation, potentiate functional disability in critical patients ${ }^{7}$. Early mobilization and rehabilitation in the course of critical illness has been proposed to improve respiratory and diaphragmatic muscle strength, attenuate functional impairments and be associated with better clinical prognosis 8,9 , suggesting a promising therapeutic intervention for patients with COVID-19.

As the effects of rehabilitation therapy on COVID-19 patients remains to be elucidated, we sought to assess the effects of early physical and pulmonary rehabilitation on the clinical and functional outcomes in severely and critically ill COVID-19 patients. The goal of this multicenter study was to provide evidence for the potential benefits of early rehabilitation therapy in these patients and suggest an option other than routine respiratory support.

\section{Methods}

\section{Patients}

We performed the descriptive, multicenter study in accordance with the Declaration of Helsinki and patients with COVID-19 were diagnosed according to the Chinese management guideline for COVID-19 (version 6.0) ${ }^{10}$. Severity of COVID-19 was defined as mild, moderate, severe and critical according to the report of the WHO-China Joint Mission on Coronavirus Disease $2019^{11}$. We screened the laboratory confirmed 
COVID-19 patients admitted to four designated hospitals in the Chongqing provincial municipality (Qianjiang central hospital of Chongqing, Chongqing three gorges central hospital, Yongchuan hospital of Chongqing medical university, and Chongqing public health medical center) from January 21 to March 15, 2020 to identify their suitability. The severely and critically ill patients admitted to the ICU with stable cardiovascular, hemodynamic, pulmonary or neuropsychological statuses were enrolled. Patients were excluded for the following reasons: dyspnea, a respiratory frequency of more than 40 beats/min, a blood oxygen saturation of less than $90 \%$, marked ventilator asynchrony, insecure airway, a systolic blood pressure (SBP) of less than 90 or more than $180 \mathrm{mmHg}$, a mean blood pressure (MAP) of less than 65 or more than $110 \mathrm{mmHg}$, a heart rate (HR) of less than 40 beats/min or more than 130 beats/min, a temperature of more than $38.5^{\circ} \mathrm{C}$, agitation, deep venous thrombosis, chronic heart failure, active myocardial ischaemia, severe ischemic or hemorrhagic stroke, severe neurodegenerative diseases, absent limbs, and less than 18 years of age.

\section{Study Design and Procedures}

Patients in the control group received the standard or usual treatment, while patients in the intervention group received physical and pulmonary rehabilitation therapies within $72 \mathrm{~h}$ of admission to the ICU. Physical and pulmonary rehabilitation therapies included (1) position management and prone-position ventilation, (2) early mobilization, (3) respiratory muscle training, (4) percussion and vibrations, (5) and stretching and physical exercises. For body position management, patients were assisted to gradually increase antigravity position until they could actively maintain a vertical position for $30 \mathrm{~min}$ at a time, three times a day. ARDS patients underwent prone-position ventilation for at least $12 \mathrm{~h}$. For early mobilization, patients began with assisted or independent mobility exercises in the supine or lateral decubitus position. If they tolerated these exercises, patients were encouraged to increase bed mobility activities and sitting balance activities. These exercises gradually progressed to transfer training, including sit-to-stand and moving from the bed to the chair repetitions, followed by stepping on the spot, and finally, walking. For respiratory muscle training, conscious patients were instructed to undergo pursed-lip and deep breathing exercises, and were trained with a commercial positive expiratory pressure device (Acapella Choice; Smiths Medical ASD, Inc. Keene, USA). Patients breathed in through Acapella Choice, held their breath for two to three seconds, breathed out slowly and smoothly for six to nine seconds, and finally performed coughs. Patients repeated these breathing exercises 10 times per session and four times a day. For percussion and vibrations, patients underwent cupped hands and high frequency chest wall oscillation to shift secretions from the peripheral airway to the central airway and enhance airway clearance and postural drainage. For stretching exercises, patients were ordered to move their limbs in flexion, extension, and rotation. The physical exercises, repeated $30 \mathrm{~min}$ a day, included passive or active axial loading exercises and activities against gravity.

The entire rehabilitation therapy progression that was dependent on patients' tolerance, medical stability, and cognitive abilities was strictly monitored. The following conditions suggested cardiovascular, hemodynamic, pulmonary, or neuropsychological instabilities that hampered the continuation of pulmonary rehabilitation therapy: dyspnea, a respiratory frequency of more than 40 beats/min, arrhythmia, a pulse oximetry of less than $88 \%$, new ventilator asynchrony, a SBP of less than 90 or more than $180 \mathrm{mmHg}$, a MAP of less than 65 or more than $110 \mathrm{mmHg}$, a HR of less than 40 or more than 130 beats/min, deterioration of conscious state, new agitation, and being physically fatigued or antagonistic.

\section{Sample and Data Collection}

Respiratory specimens were collected at admission to detect the presence of SARS-CoV-2 by real-time reverse-transcriptase polymerasechain-reaction (RT-PCR) assay in designated authoritative laboratories of local centers for disease control and prevention. Cytokines and lymphocyte subsets in peripheral blood were measured by fluorescence-labeled flow cytometry (Beckman, Cell Lab Quanta SC). Spirometry and pulmonary diffusion capacity tests (MasterScreen, Hoechberg, Germany) were performed following the American Thoracic Society and European Respiratory Society's guidelines, on the day of discharge, as well as one month and three months after hospital discharge. The epidemiological, demographic, clinical, laboratory, radiological, treatment and outcomes data from patients' medical and nursing records were obtained and analyzed. The date of disease onset was defined as the day when the symptoms were noticed. Lastly, we entered the data into a computerized database and two physicians verified this data.

\section{Outcomes}

PCR-confirmed negative patients were evaluated at hospital discharge, and one month as well as three months after hospital discharge in the local ambulatory clinic. The chosen primary outcomes were 28-day, ICU, and hospital mortality rates, lengths of stay in the ICU and hospital, duration of mechanical ventilation, ventilator-free days, complications, and adverse occurrences during hospital stays. We defined the secondary outcomes at hospital discharge and during the three-month follow-up as follows. To assess pulmonary function, we measured the forced expiratory volume within one second (FEV1), forced vital capacity (FVC), total lung capacity (TLC), and carbon monoxide diffusion capacity (DLCO). For independent functional status, we evaluated activities of daily living (ADL) with the Barthel index score that covers 10 subtest items (feeding, moving from wheelchair to bed and return, grooming, getting on/off the toilet, bathing, walking, stair climbing, dressing, bowel and bladder continence) ranging from 0 to 100. We also used the Functional Independence Measure (FIM) 
that contains 18 items (13 for motor ADL and five for cognitive ADL) ranging from 1 (requiring total assistance) to 7 (completely independent). We estimated muscle strength using the Medical Research Council (MRC) score and incidences of ICU-acquired weakness (ICU-AW). Exercise capacity was evaluated through the standard six-minute walk test (6MWT), using the saturated pulse oximetry to monitor vital signs and percutaneous oxygen saturation. Lastly, health-related quality of life was assessed by St. George's Respiratory Questionnaire (SGRQ) that includes three components measuring patients' impaired health and perceived well-being. The scores range from 0 to 100 , with higher scores indicating more limitations.

\section{Definitions}

Fever was defined as an axillary temperature of $37.3^{\circ} \mathrm{C}$ or higher. ARDS was defined and classified according to the Berlin definition ${ }^{12}$. Sepsis were defined according to the 2016 Third International Consensus Definition for Sepsis and Septic Shock ${ }^{13}$. Acute kidney injury was diagnosed according to the KDIGO clinical practice guidelines ${ }^{14}$. Cardiac injury was diagnosed if the serum concentration of cardiac biomarker (e.g., high-sensitivity cardiac troponin I, hsTnl) was above the 99th percentile upper reference limit (>28 pg/mL) or if new abnormalities were shown in the electrocardiography. Hypoproteinaemia was defined as a serum albumin of less than $25 \mathrm{~g} / \mathrm{L}$. ICU-AW referred to cooperative patients who had a MRC score of less than 48 out of 60 points, after the assessment of the muscle strength in 12 regions on both sides of the upper and lower limbs.

\section{Statistical Analysis}

Continuous variables were expressed as the means and standard deviations (S.D.) or medians and interquartile ranges (IQR) as appropriate. Categorical variables were presented as the counts and percentages. We performed comparisons with independent group t-tests between continuous variables of two groups when the data were normally distributed; otherwise, we used the Mann-Whitney $U$ test. Categorical variables' proportions were compared using the Chi-square test or Fisher's exact test. The repeated measurements of pulmonary function test and scores (Barthel Index, FIM, MRC, SGRQ, and 6MWT) at different time points were compared with one-way or two-way analysis of variance followed by Turkey post-hoc tests. All the tests were two-sided and a value of $p<0.05$ was considered statistically significant. Finally, we performed the data analyses using GraphPad Prism 7.0 software.

\section{Results}

\section{Demographics and Clinical Characteristics}

From January 21 to March 15, 2020, we screened 427 laboratory-confirmed patients. We excluded 54 patients, due to crucial data incompleteness in the original reports, and enrolled 89 severely and critically ill patients from the ICU. Among them, we excluded three patients who suffered from acute myocardial infarction or cardiogenic shock, three who were unable to complete the rehabilitation therapies, and another three patients who were unable to complete the pulmonary function test. Thus, a total of 80 severely and critically ill COVID-19 patients from four designated-hospitals were included in this study, of whom 43 patients completed the physical and pulmonary rehabilitation therapies were assigned to the intervention group, and the remaining 37 patients were assigned to the control group.

Table 1 lists their baseline demographics and clinical characteristics. We observed no significant differences in age, gender, comorbidities, independent functional status, and disease severity between the two groups. On admission, the typical signs and symptoms included fever, cough, and myalgia or arthralgia. Notably, dyspnea was a common symptom in severely and critically ill COVID-19 patients. There were no significant differences in abnormal physical examination signs on admission. 
Table 1

Demographics and baseline clinical characteristics of the COVID-19 patients in the study

\begin{tabular}{|c|c|c|c|c|}
\hline Clinical characteristics & $\begin{array}{l}\text { All patients } \\
(n=80)\end{array}$ & $\begin{array}{l}\text { Intervention } \\
(n=43)\end{array}$ & $\begin{array}{l}\text { Control } \\
(n=37)\end{array}$ & $p$ value \\
\hline Age, Median (IQR)-yrs & $67(52.75-75.25)$ & $68(52-74.5)$ & $66(57-77)$ & 0.889 \\
\hline \multicolumn{5}{|l|}{ Age groups-No. (\%) } \\
\hline$\geq 60$ yrs & $55(68.75)$ & $29(67.44)$ & $26(70.27)$ & 0.7855 \\
\hline Male sex- No. (\%) & $48(60)$ & $26(60.47)$ & $22(59.46)$ & 0.9271 \\
\hline APACHE II score-median (IQR) & $18(14-22)$ & $17(13.5-21.5)$ & $20(16-23)$ & 0.0939 \\
\hline CURB-65 score & $3.5(3-4)$ & $4(3-5)$ & $3(3-4)$ & 0.2458 \\
\hline Barthel Index Score-median (IQR) & $75(66.75-80)$ & $75(68-87)$ & $75(65-79)$ & 0.0561 \\
\hline \multicolumn{5}{|l|}{ Smoking history- No. (\%) } \\
\hline Smokers & 39 (48.75) & $24(55.81)$ & $15(40.54)$ & 0.1730 \\
\hline \multicolumn{5}{|l|}{ Comorbidities-No. (\%) } \\
\hline Overweight or obesity & $17(21.25)$ & $9(20.93)$ & $8(21.62)$ & 0.9399 \\
\hline Diabetes or impaired fasting glucose & $14(17.5)$ & $8(18.6)$ & $6(16.22)$ & 0.7792 \\
\hline Hypertension & $16(20)$ & $10(23.26)$ & $6(16.22)$ & 0.4326 \\
\hline Respiratory system disease & $11(13.75)$ & $6(13.95)$ & $5(13.51)$ & 0.9546 \\
\hline Cardiovascular and cerebrovascular diseases & $12(15)$ & $6(13.95)$ & $6(16.22)$ & 0.7775 \\
\hline \multicolumn{5}{|l|}{ Symptoms and signs-No. (\%) } \\
\hline Fever on admission & $60(75)$ & $33(76.74)$ & $27(72.97)$ & 0.6977 \\
\hline Temperature on admission $\left({ }^{\circ} \mathrm{C}\right)$ & $37.5(37.3-38)$ & $37.5(37.3-37.9)$ & $37.6(37.1-38.4)$ & 0.5717 \\
\hline $37.3-38$ & $40(50)$ & $23(53.49)$ & $17(45.95)$ & 0.5011 \\
\hline$>38$ & $20(25)$ & $10(23.26)$ & $10(27.03)$ & 0.6977 \\
\hline Cough & $56(70)$ & $34(79.07)$ & $22(59.46)$ & 0.0563 \\
\hline Myalgia or arthralgia & $33(41.25)$ & $14(32.56)$ & 19 (51.35) & 0.0887 \\
\hline Diarrhea & $6(7.5)$ & $2(4.65)$ & $4(10.81)$ & 0.2970 \\
\hline Pharyngalgia & $15(18.75)$ & $6(13.95)$ & $9(24.32)$ & 0.2360 \\
\hline Dyspnea & $35(43.75)$ & $20(46.51)$ & $15(40.54)$ & 0.5914 \\
\hline Confusion & $10(12.5)$ & $5(11.63)$ & $5(13.51)$ & 0.7993 \\
\hline Nausea or vomiting & $4(5)$ & $3(6.98)$ & $1(2.7)$ & 0.3818 \\
\hline Anorexia & $31(38.75)$ & $19(44.19)$ & $12(32.43)$ & 0.2820 \\
\hline \multicolumn{5}{|l|}{ Signs-No. (\%) } \\
\hline Throat congestion & $14(17.5)$ & $6(16.22)$ & $8(18.6)$ & 0.7792 \\
\hline Enlargement of lymph nodes & $3(3.75)$ & $2(4.55)$ & $1(2.7)$ & 0.6618 \\
\hline Rales & $12(15)$ & $7(16.28)$ & $5(13.51)$ & 0.7298 \\
\hline Wheezes & $9(11.25)$ & $5(11.63)$ & $4(10.81)$ & 0.9082 \\
\hline \multicolumn{5}{|l|}{ Onset of symptom to-median (IQR) } \\
\hline Laboratory diagnosis & $3(2-5)$ & $3(2-5)$ & $3(2-4)$ & 0.3396 \\
\hline
\end{tabular}




\begin{tabular}{|lllll|}
\hline Clinical characteristics & $\begin{array}{l}\text { All patients } \\
(\mathbf{n}=\mathbf{8 0})\end{array}$ & $\begin{array}{l}\text { Intervention } \\
(\mathbf{n}=\mathbf{4 3})\end{array}$ & $\begin{array}{l}\text { Control } \\
(\mathbf{n}=\mathbf{3 7})\end{array}$ & $\boldsymbol{p}$ value \\
\hline Hospital admission & $3(2-5)$ & $4(2-5)$ & $3(1-4)$ & 0.0561 \\
ICU admission & $4(3-6)$ & $5(3.5-7.5)$ & $4(3-5)$ & 0.3523 \\
\hline
\end{tabular}

\section{Laboratory and Radiologic Results}

As listed in Supplementary Table 1, no obvious differences were observed in the major laboratory and radiologic results on admission. Most patients in our study had lymphopenia with decreases in CD4 positive T cell counts. The CD8 positive T cell levels were lower than normal in close to half of the patients. Additionally, declines in albumin and elevation in the D-dimer were common in the severely and critically ill patients. Regarding the infectious index, $70.0 \%$ and $56.25 \%$ of patients had higher levels of C-reactive protein and procalcitonin, respectively. Furthermore, we observed high levels of IL-6, TNF-a, and IL-17A in $65.0 \%, 55.0 \%$, and $51.25 \%$ of cases, respectively. As for radiologic examinations, most patients had pneumonia with ground-glass opacity or patchy shadowing, and consolidation or fibrous stripes-like shadows were observed in $43.75 \%$ of patients.

\section{Treatment Strategies}

All severe and critically ill patients in our study were treated in the ICU with isolated wards. Supplementary Table 2 illustrates the range of treatments. Antiviral therapies and empirical antibiotic treatments were administrated to all severely and critically ill COVID-19 patients in our study, while $76.25 \%$ of patients without obvious immune deficiencies received systemic glucocorticoid therapy, usually methylprednisolone $1-2 \mathrm{mg} / \mathrm{kg}$ for three to five days. A total of 57 patients received immunopotentiators (thymalfasin $1.6 \mathrm{mg} / \mathrm{day}$ ) or immunoglobulin treatments (human $\mathrm{y}$-globulin, $200-300 \mathrm{mg} / \mathrm{kg}$ for three to five days). Invasive mechanical ventilation, non-invasive mechanical ventilation, and high-flow nasal cannula (HFNC) oxygen therapy was adopted in $11.25 \%, 60 \%$, and $58.75 \%$ of patients with respiratory failure, respectively. Compared to the intervention group, patients in the control group received more HFNC therapy. Most patients received traditional Chinese medicine and anticoagulation therapy, and only a few received extracorporeal membrane oxygenation (ECMO), kidney replacements, and convalescent plasma therapy.

\section{Clinical Outcomes}

As shown in Table 2, there were no significant differences in the ICU and hospital mortality between the intervention and control groups. A 78 year old female in the control group died from cardiac arrest three days after hospital discharge, potentially due to an acute pulmonary thromboembolism. Thus, the 28 -day mortality in our study is $5 \%$, with no significant differences between the two groups. We also found no significant differences in the median length of stay in the ICU and hospital stay for patients between the intervention and control groups. As for mechanical ventilation therapies, we found that the median duration of non-invasive mechanical ventilation and HFNC significantly decreased in the intervention group compared to the control group, and early rehabilitation enhanced the number of ventilator-free days. We also noted no significant difference in the median duration of invasive mechanical ventilation. In our study, early rehabilitation therapy did not increase the rate of adverse events, and incidences of deep vein thrombosis were lower in the intervention group than in the control group. Common complications were ARDS, acute kidney injury, shock, and acute cardiac injury, with no obvious differences between the two groups. 
Table 2

Primary outcomes of the study

\begin{tabular}{|c|c|c|c|c|}
\hline & $\begin{array}{l}\text { All patients } \\
(n=80)\end{array}$ & $\begin{array}{l}\text { Intervention } \\
(n=43)\end{array}$ & $\begin{array}{l}\text { Control } \\
(n=37)\end{array}$ & $p$ value \\
\hline Mortality-No. (\%) & $4(5)$ & $2(4.65)$ & $2(5.41)$ & 0.8773 \\
\hline 28-days & $3(3.75)$ & $1(2.33)$ & $2(5.41)$ & 0.4697 \\
\hline ICU & $3(3.75)$ & $1(2.33)$ & $2(5.41)$ & 0.4697 \\
\hline \multicolumn{5}{|l|}{ hospital } \\
\hline \multicolumn{5}{|l|}{ Length of stay-median (IQR), d } \\
\hline in ICU & $11(8-14)$ & $11(7-13)$ & $11(8-14.5)$ & 0.2221 \\
\hline in hospital & $15(12-18)$ & $15(11-17.75)$ & $16(13.5-18)$ & 0.2482 \\
\hline \multicolumn{5}{|c|}{ Duration of mechanical ventilation- median (IQR), $d$} \\
\hline Invasive & $5(3-6)$ & $5(3-5)$ & $5(3.75-6.5)$ & 0.5397 \\
\hline Non-invasive & $12(10-14)$ & $10(9-13)$ & $14(12-16)$ & 0.0164 \\
\hline HFNC & $7(4-10)$ & $4.5(3-7)$ & $9(7-11.5)$ & 0.0002 \\
\hline Number of ventilatory-free days-median (IQR) & $5(2-5)$ & $5(3-6)$ & $4(2-5)$ & 0.0083 \\
\hline Adverse events-No. (\%) & $5(6.25)$ & $2(4.65)$ & $3(8.11)$ & 0.5242 \\
\hline \multicolumn{5}{|l|}{ Arrhythmia } \\
\hline Myocardial infarction & $3(3.75)$ & $0(0)$ & $3(8.11)$ & 0.0570 \\
\hline Deep vein thrombosis & $9(11.25)$ & $2(4.65)$ & $7(18.92)$ & 0.0440 \\
\hline Decannulation of catheter & $3(3.75)$ & $2(4.65)$ & $1(2.7)$ & 0.6474 \\
\hline Pneumothorax & $3(3.75)$ & $2(4.65)$ & $1(2.7)$ & 0.6474 \\
\hline Pulmonary embolism & $4(5)$ & $2(4.65)$ & $2(5.41)$ & 0.8773 \\
\hline \multicolumn{5}{|l|}{ Complications-No. (\%) } \\
\hline ARDS & $42(56.25)$ & $20(46.51)$ & $22(59.46)$ & 0.2476 \\
\hline Shock & $7(8.75)$ & $2(4.65)$ & $5(13.51)$ & 0.1619 \\
\hline Acute cardiac injury & $3(3.75)$ & $0(0)$ & $3(8.11)$ & 0.0570 \\
\hline Acute kidney injury & $11(13.75)$ & $6(13.95)$ & $5(13.51)$ & 0.9546 \\
\hline DIC & $3(3.75)$ & $1(2.33)$ & $2(5.41)$ & 0.4697 \\
\hline
\end{tabular}

\section{Pulmonary Function Tests}

On the day of discharge, the room air oxygen saturation at rest was normal in all cases and no significant difference was observed between two groups. As shown in Table 3-5, we noted pulmonary function abnormalities in the percentage of observed FVC related to its predicted values (FVC \% predicted) in 36 cases, the percentage of observed FEV1 related to its predicted values (FEV1\% predicted) in 37 patients, and the FEV1/FVC ratio (FEV1/FVC \%) in 24 cases at hospital discharge, that significantly improved after three months of recovery. Besides, as shown in Table 3-5 and Fig. 1, no obvious differences in these ventilatory parameters were found between intervention and control groups at hospital discharge, as well as one month and three months after hospital discharge (all $p>0.05$ ). 
Table 3

Secondary outcomes of the study at hospital discharge

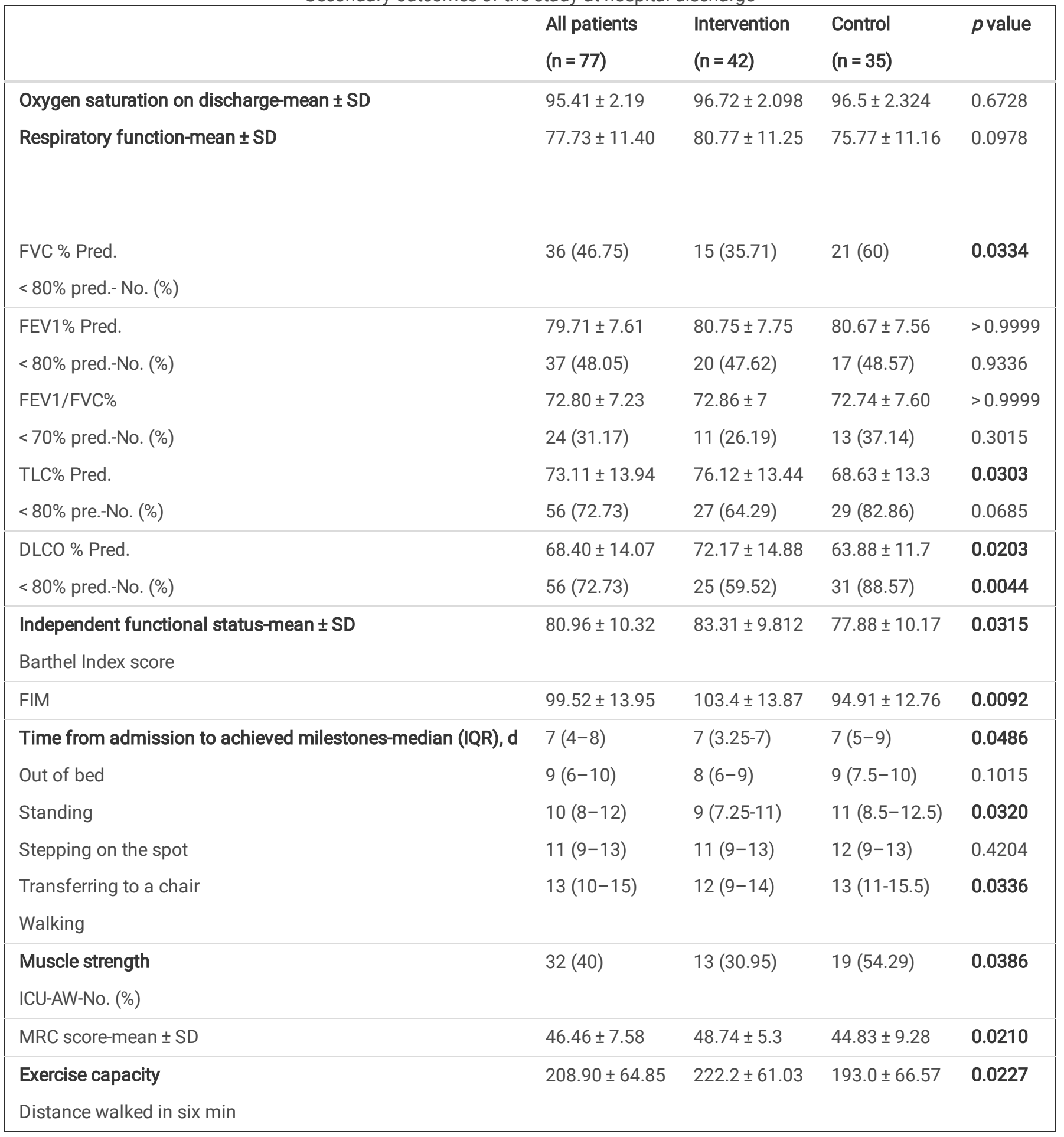


Table 4

Secondary outcomes of the study: one month after hospital discharge

\begin{tabular}{|c|c|c|c|c|}
\hline & $\begin{array}{l}\text { All patients } \\
(n=76)\end{array}$ & $\begin{array}{l}\text { Intervention } \\
(n=42)\end{array}$ & $\begin{array}{l}\text { Control } \\
(n=34)\end{array}$ & $p$ value \\
\hline Respiratory function-mean \pm SD & $86.8 \pm 9.66$ & $89.08 \pm 10.2$ & $86.24 \pm 8.847$ & 0.7105 \\
\hline $\begin{array}{l}\text { FVC \% Pred. } \\
<80 \% \text { pred.-No. (\%) }\end{array}$ & \multicolumn{3}{|c|}{$<80 \%$ pred.-No. (\%) } & 0.3020 \\
\hline FEV1\% Pred. & $88.39 \pm 6.25$ & $89.96 \pm 7.165$ & $88.87 \pm 4.941$ & $>0.9999$ \\
\hline$<80 \%$ pred..-No. (\%) & $5(6.58)$ & $4(9.52)$ & $1(2.94)$ & 0.2498 \\
\hline FEV1/FVC\% & $81.55 \pm 8.54$ & $82.4 \pm 8.139$ & $82.65 \pm 9.126$ & $>0.9999$ \\
\hline$<70 \%$ pred.-No. (\%) & $7(9.21)$ & $4(9.52)$ & $3(8.82)$ & 0.9164 \\
\hline TLC\% Pred. & $82.05 \pm 13.63$ & $85.29 \pm 13.02$ & $78.03 \pm 13.48$ & 0.0400 \\
\hline < 80\% pred.-No. (\%) & $29(38.16)$ & $10(23.81)$ & 19 (55.88) & 0.0042 \\
\hline DLCO \% Pred. & $71.57 \pm 14.84$ & $75.99 \pm 15.02$ & $67.71 \pm 13.13$ & 0.0218 \\
\hline$<80 \%$ pred.-No. $(\%)$ & $47(61.84)$ & $20(47.62)$ & $27(79.41)$ & 0.0046 \\
\hline $\begin{array}{l}\text { Independent functional status } \\
\text { Barthel Index score-mean } \pm \text { SD }\end{array}$ & \multicolumn{3}{|c|}{ Barthel Index score-mean \pm SD } & 0.0249 \\
\hline FIM-mean \pm SD & $100.28 \pm 13.3$ & $103.50 \pm 12.09$ & $96.24 \pm 13.78$ & 0.0353 \\
\hline Muscle strength & $26(34.21)$ & $8(19.05)$ & $18(52.94)$ & 0.0020 \\
\hline ICU-AW-No. (\%) & $49.95 \pm 7.36$ & $52.24 \pm 5.314$ & $48.32 \pm 6.535$ & 0.0219 \\
\hline \multicolumn{5}{|l|}{ MRC score-mean \pm SD } \\
\hline Exercise capacity & $280.08 \pm 37.82$ & $293 \pm 31.6$ & $264.1 \pm 39.15$ & 0.0262 \\
\hline Distance walked in six min-mean \pm SD & $51.74 \pm 9.56$ & $49.6 \pm 9.045$ & $55.62 \pm 9.24$ & 0.0089 \\
\hline \multicolumn{5}{|l|}{ Health-related quality of life } \\
\hline SGRQ score- mean \pm SD & & & & \\
\hline
\end{tabular}


Table 5

Secondary outcomes of the study: three months after hospital discharge

\begin{tabular}{|c|c|c|c|c|}
\hline & $\begin{array}{l}\text { All patients } \\
(n=76)\end{array}$ & $\begin{array}{l}\text { Intervention } \\
(n=42)\end{array}$ & $\begin{array}{l}\text { Control } \\
(n=34)\end{array}$ & $p$ value \\
\hline Respiratory function-mean \pm SD & $87.73 \pm 9.61$ & $90.14 \pm 10.19$ & $87.05 \pm 8.679$ & 0.5661 \\
\hline FVC \% Pred. & $12(15.79)$ & $5(11.9)$ & $7(20.59)$ & 0.3020 \\
\hline \multicolumn{5}{|l|}{ < 80\% pred.-No. (\%) } \\
\hline FEV1\% Pred. & $90.08 \pm 6.82$ & $92.12 \pm 7.122$ & $90.01 \pm 6.415$ & 0.5675 \\
\hline < 80\% pred., No. (\%) & $6(7.89)$ & $3(7.14)$ & $3(8.82)$ & 0.7870 \\
\hline FEV1/FVC\% & $81.55 \pm 8.54$ & $84.07 \pm 8.259$ & $84.49 \pm 9.496$ & $>0.9999$ \\
\hline$<70 \%$ pred.-No. (\%) & $8(10.53)$ & 5 (11.9) & $3(8.82)$ & 0.6634 \\
\hline TLC\% pred. & $84.66 \pm 10.52$ & $87 \pm 11.25$ & $83.86 \pm 10.9$ & 0.8451 \\
\hline$<80 \%$ pred.-No. (\%) & $29(22.37)$ & $11(16.67)$ & $18(29.41)$ & 0.1849 \\
\hline \multirow[t]{2}{*}{ DLCO \% Pred. } & $79.26 \pm 12.59$ & $84.45 \pm 11.73$ & $76.52 \pm 12.1$ & 0.0303 \\
\hline & & $11(26.19)$ & $18(52.94)$ & 0.0170 \\
\hline$<80 \%$ pred.-No. (\%) & $29(38.16)$ & & & \\
\hline Independent functional status & $86.26 \pm 7.7$ & $86.57 \pm 8.13$ & $85.88 \pm 7.24$ & $>0.9999$ \\
\hline \multicolumn{5}{|l|}{ Barthel Index score-mean \pm SD } \\
\hline FIM-mean \pm SD & $103.82 \pm 10.71$ & $104.5 \pm 11.35$ & $103 \pm 9.97$ & $>0.9999$ \\
\hline Muscle strength & $14(18.42)$ & 5 (11.9) & $9(26.47)$ & 0.1034 \\
\hline ICU-AW-No. (\%) & $50.67 \pm 5.64$ & $52.29 \pm 3.59$ & $48.68 \pm 6.99$ & 0.0402 \\
\hline \multicolumn{5}{|l|}{ MRC score-mean \pm SD } \\
\hline Exercise capacity & $357.57 \pm 56.98$ & $376.8 \pm 33.07$ & $341.3 \pm 42.37$ & 0.0040 \\
\hline Distance walked in six min-mean \pm SD & $46.36 \pm 9.52$ & $43.79 \pm 8.697$ & $50.62 \pm 9.24$ & 0.0026 \\
\hline \multicolumn{5}{|l|}{ Health-related quality of life } \\
\hline SGRQ score-mean \pm SD & & & & \\
\hline
\end{tabular}

As shown in Table 3-5, most discharged patients had obvious impairments in lung volume and diffusing capacity at hospital discharge, as manifested by the abnormalities in the percentage of observed TLC related to its predicted values (TLC \% predicted) in 56 cases, and the percentage of observed DLCO related to its predicted values (DLCO \% predicted) in 56 cases. Figure 1 illustrated that, from hospital discharge to one month after, TLC \% predicted and DLCO \% predicted improved from $73.11-82.05 \%(p<0.0001)$ and from $68.40-71.57 \%(p$ $=0.3468)$, respectively. By three months, these median values significantly improved to $84.66 \%(p<0.0001)$ and $79.26 \%(p<0.0001)$, respectively. At hospital discharge, TLC \% predicted and DLCO \% predicted for patients in the intervention group were higher than those in the control group, indicating lung volume and diffusing capacity improvements stemming from the early physical and pulmonary rehabilitation therapy. One month after discharge, these improvements were still present. However, three months after discharge, although the DLCO \% predicted of patients in the intervention group was still higher, the TLC \% predicted exhibited a notable, but statistically insignificant, enhancement between the two groups (Fig. 1 and Table 3-5).

\section{Independent Functional Status}

Barthel Index score and FIM of patients did not show significant alternations during the one-month follow-up after hospital discharge (80.96 \pm 10.32 vs $82.25 \pm 10.55, p=0.6849 ; 99.52 \pm 13.95$ vs $100.28 \pm 13.30, p=0.9277)$. The Barthel Index score significantly improved three months after hospital discharge, compared to the first month ( $82.25 \pm 10.55$ vs $86.26 \pm 7.70, p=0.0289)$, while the FIM scale showed no significant changes (100.28 \pm 13.30 vs $103.82 \pm 10.71, p=0.2024)$ (Fig. 2). At hospital discharge, patients in the intervention group had 
significantly higher Barthel Index scores and FIM than those in the control group, indicating that severe COVID-19 patients benefited from early rehabilitation, as it helped them return to independent functional status. These beneficial effects lasted for one month after discharge, as evidenced by the higher Barthel Index scores and FIM in the intervention group than in the control group. There was no further significant improvement at three months after discharge (Fig. 2, Table 3-5). Additionally, at hospital discharge, patients in the intervention group needed less time to achieve several activity milestones, such as getting out of bed, stepping on the spot, and walking (Table 3 ).

\section{Muscle Strength}

As demonstrated in Fig. 2, compared to the value at hospital discharge, the MRC score significantly enhanced one month after hospital discharge $(46.46 \pm 7.58$ vs $49.95 \pm 7.36, p=0.0058)$, with no further significant improvements after three months (49.95 \pm 7.36 vs $50.67 \pm$ $5.64, p=0.7973)$. Patients in the intervention group had a significantly higher MRC score at hospital discharge, as well as one month and three months after hospital discharge. Less ICU-AW was noted in the intervention group than in the control group at hospital discharge and one month after discharge. At three months after discharge, we found a decreasing, but statistically insignificant trend in ICU-AW for the control group (Fig. 2, Table 3-5).

\section{Exercise Capacity}

Walking distance within 6 minutes significantly increased one month after hospital discharge compared to right after discharge (208.90 \pm 64.85 vs $280.08 \pm 37.82, p<0.0001)$, with further significant improvements after three months $(280.08 \pm 37.82$ vs $357.57 \pm 56.98, p<0.0001)$ (Fig. 2). Patients in the intervention group had a significantly greater walking distance in six minutes at hospital discharge, as well as one month and three months after discharge (Fig. 2, Table 3-5).

\section{Health-Related Quality of Life}

The SGRQ score at one month after hospital discharge was higher than at three months after hospital discharge (51.74 \pm 9.56 vs $46.36 \pm$ $9.52, p=0.0007$ ). This score was lower in the intervention group than in the control group at one month and three months after hospital discharge (Table 4, 5), indicating that early rehabilitation accelerated the improvement of life quality in severely and critically ill COVID-19 patients.

\section{Discussion}

Our present study demonstrated that, although no significant alternations in 28-day, ICU, and hospital mortality rates, as well as patients' lengths of stay in hospital and ICU were found, early physical and pulmonary rehabilitation in severely and critically ill patients with COVID19 increased the duration of ventilator-free days during hospitalization, with no increased adverse events and complications. Additionally, early rehabilitation in severely ill COVID-19 patients enhanced the recovery of lung volume and diffusing capacity, accelerated patients' return to independent functional status, decreased ICU-AW incidences, and increased muscle strength and exercise capacity at hospital discharge and one month after discharge. The improvements in lung diffusing capacity, muscle strength, life quality, and exercise capacity remained significant up to three months after hospital discharge. Therefore, the implementation of early physical and pulmonary rehabilitation was safe and effective for severely and critically ill patients with COVID-19 to promote their functional, physical and psychological recovery.

Skeletal muscle mass is known to be an important determinant factor for critical patients to overcome diseases in the ICU ${ }^{7}$. Loss of skeletal muscle mass, quality, and strength in the ICU is an independent mortality risk factor for critically ill patients, and muscle weakness affecting the limbs and respiratory muscles during ICU hospitalization is associated with poor outcomes, such as delayed recovery, hindered weaning from mechanical ventilation, decreased life quality, and increased financial costs ${ }^{15,16}$. Accumulated evidence has revealed that early ICU rehabilitation, including pulmonary rehabilitation to improve respiratory function and remove airway secretions, and physical rehabilitation to attenuate muscle weakness, has positive impacts on critically ill patients' prognoses ${ }^{8,9,17-19}$. Therefore, early rehabilitation in the ICU is supposed to improve the clinical outcomes and functional recovery of severe COVID-19 patients. However, to our knowledge, a limited number of studies have reported the impacts of early physical and pulmonary rehabilitation on COVID-19 patients, especially for severe and critical cases. For example, one case report shared the early pulmonary rehabilitation experiences of a 41-year-old severe patient with SARSCoV-2 pneumonia ${ }^{20}$. Another study found that a six-week respiratory rehabilitation program produced positive impacts on COVID-19 patients aged over 65 by improving respiratory functions, quality of life, and exercise endurance, as well as relieving anxiety ${ }^{21}$. However, no study has systematically assessed the effects of early physical and pulmonary rehabilitation on severe COVID-19 patients. In our present study, we found that implementing early rehabilitation in severe and critical patients with COVID-19 increased the amount of ventilator-free days during hospitalization, largely attributed to the removal of airway secretions and amelioration of respiratory failure. These results were 
consistent with most previous reports concerning the beneficial impacts of early physical medicine and rehabilitation on critically ill patients in the $\mathrm{ICU}^{22}$.

Previous study has found that lung functional impairments in the recovered patients with SARS-coronavirus pneumonia were common and could last for months or even years, among which impaired diffusing capacity and defected lung volume were the most common abnormalities $^{1}$. In our present study, we witnessed varying degrees of declining DLCO, TLC, FEV1, FVC, and FEV1/FVC levels in the severe and critical COVID-19 patients at hospital discharge, consistent with a recent report revealing the diffusion capacity impairments and restrictive ventilatory defects in COVID-19 patients at hospital discharge ${ }^{23}$. Histological examinations of the lung tissue in COVID-19 patients confirmed the diffuse alveolar damages with hyaline membrane formation and fibromyxoid exudates ${ }^{24}$. Follow-up study lasting for one year found that the persistent functional limitation in the survived ARDS patients was largely a result of muscle wasting and weakness, due to their immobilization during hospitalization ${ }^{6}$. Thus, pulmonary alveolar edemas, interstitial fibrous proliferation, and muscle wasting collectively resulted in impaired lung function. Previous study found that respiratory rehabilitation could clearly enhance improvements in the respiratory functions of elderly COVID-19 patients ${ }^{21}$. However, we only found significant promotions in lung volume and diffusing capacity, potentially due to the differences in our patients' disease severity, baseline comorbidities, and smoking history.

In our current study, the interventions of early rehabilitation therapy significantly improved independence functional outcomes at hospital discharge and one month after discharge, supporting the benefits and feasibility of these interventions on the restoration of functional independence. Our results are consistent with previous findings demonstrating that whole-body rehabilitation in the earliest days of critical illness result in better functional outcomes, as reflected by higher Barthel Index scores, more independent ADLs, and greater unassisted walking distance at hospital discharge ${ }^{9}$. After three months, the independence functions improved for all patients and the beneficial effects of rehabilitation therapy subsided. A variety of improvements in muscles, nerves, metabolism immunity, and psychological states may explain the long-term recovery.

The adverse impacts of skeletal muscle immobility are well-recognized, as individuals show skeletal muscle atrophic signs if immobilized for more than 72 hours, and loss of muscle mass and strength can be noted even in well-nourished healthy older adults within 10 days of bed rest ${ }^{25,26}$. Therefore, physical rehabilitation in the ICU for severe COVID-19 patients, especially those combined with ARDS, is supposed to be an important intervention to preserve muscle architecture, improve muscle strength, and decrease ICU-AW. In present study, we confirmed that early physical rehabilitation results in improvements in muscle strength and exercise capacity in the three-month follow-up, with decreases in ICU-AW incidences at hospital discharge and one month after discharge. Impaired muscle function may explain the compromised quality of life. Thus, we postulate that the observed improvements in life quality and physical function may partially be due to the remission of muscle weakness. These results are consistent with most reports supporting the beneficial effects of early mobilization and rehabilitation on greater muscle strength in critically ill patients ${ }^{17,22}$. However, one meta-analysis found that early mobilization and rehabilitation had no significant effects on muscle strength, quality of life, and ICU-AW incidences, perhaps due to the inadequate inclusion of published randomized controlled trials (RCT) ${ }^{27}$. As for the mechanisms of rehabilitation training on these benefits, in addition to conventional concepts, skeletal muscles are now considered as important endocrine tissues that secrete various bioactive molecules, called myokines, that act in an auto-, para-, and endocrine manner to regulate the physiological function of target tissues and contribute to the progression of diverse diseases ${ }^{28,29}$. Rehabilitation training is speculated to protect target organs against disorders through the regulation of myokines homoeostasis, but further studies are needed to explore the implications and underlying mechanisms of myokines as related to the beneficial effects of rehabilitation therapy on severe and critical COVID-19 patients.

Despite this study's numerous strengths, we do have several limitations deserving consideration. Firstly, the sample size was relatively small with few non-survivor cases, limiting our sub-group or regression analysis. Secondly, as COVID-19 is a world public health emergency and there are insufficient clinical experience on rehabilitation therapy for severe COVID-19 cases, we varied the therapy's intensity for both the intervention and control groups, according to patients' medical stability and tolerance. Thus, we are currently conducting a retrospective study, rather than an RCT study. Thirdly, subsequent at-home rehabilitation training may have potentially biased our results and affected the outcomes.

\section{Conclusion}

In this study, we focused on implementing an early rehabilitation therapy for severely and critically ill patients with COVID-19. Early rehabilitation increased the duration of ventilator-free days during hospitalization, with no increased adverse events and complications. Furthermore, early rehabilitation enhanced pulmonary function abnormality recovery, accelerated the process of returning to independent functional status, decreased ICU-AW incidences, and increased muscle strength and exercise capacity at hospital discharge and one month after hospital discharge. The improvements in lung diffusing capacity, muscle strength, life quality, and exercise capacity remained

Page 12/17 
significant at the three-month follow-up. Further, double-blind RCT and long-term studies with larger sample sizes are required to address the full impact of early rehabilitation therapy on COVID-19 patients.

\section{Abbreviations}

ICU: intensive care unit

SARS: severe acute respiratory syndrome

ARDS: acute respiratory distress syndrome

SBP: systolic blood pressure

MAP: mean blood pressure

HR: heart rate

RT-PCR: real-time reverse-transcriptase polymerase-chain-reaction

FEV1: forced expiratory volume within one second

FVC: forced vital capacity

TLC: total lung capacity

DLCO: carbon monoxide diffusion capacity

ADL: activities of daily living

FIM: Functional Independence Measure

MRC: Medical Research Council

ICU-AW: ICU-acquired weakness

6MWT: six-minute walk test

SGRQ: St. George's Respiratory Questionnaire

hsTnl: high-sensitivity cardiac troponin I

S.D.: standard deviations

IQR: medians and interquartile ranges

HFNC: high-flow nasal cannula

ECMO: extracorporeal membrane oxygenation

RCT: randomized controlled trials

\section{Declarations}

\section{Ethics approval and consent to participate}

The institutional ethics boards of the second affiliated hospital of Chongqing medical university (No.2020-09), the Chongqing public health medical center (No.2020-015-01-KY), the Chongqing three gorges central hospital (No.2020-13), the Yongchuan hospital of Chongqing medical university (NO.2020-18), and the Qianjiang central hospital of Chongqing (No.2020-07) all approved the study's design and procedure. Informed consent was yielded, due to the anonymous data analysis obtained from a retrospective study.

\section{Consent for publication}


Not applicable

\section{Availability of data and materials}

The datasets analyzed during the current study are available from the corresponding author on reasonable request.

\section{Competing interests}

The authors declare that they have no known competing financial interests or personal relationships that could influence the work reported in this paper.

\section{Funding}

This study was supported by the Natural Science Foundation of Chongqing, China (Grant NO. cstc2020jscx-fyzx0230), the Emergency Foundation for Novel Coronavirus Pneumonia of Chongqing Health Committee, China (Grant NO. 2020NCPZX19), and the National Natural Science Foundation for Young Scholars of China (Grant NO. 81800083).

\section{Authors' contributions}

Daoxin Wang and Di Qi designed the research; Di Qi, Xiaofeng Yan, Jianglin Xiang, Junnan Peng, Xumao Tang, Jin Tong, Longhua Feng, Jing Yuan, Yuyan Song collected the data; Di Qi, Junnan Peng, Qian Yu, Xumao Tang, Jin Tong performed data analysis; Di Qi performed manuscript writing; Daoxin Wang directed the study and reviewed the manuscript.

\section{Acknowledgements}

We thank all the medical staff who participated in treating the patients. Also, we deeply thank all the patients enrolled in this study.

\section{References}

1. Hui DS, Joynt GM, Wong KT, et al. Impact of severe acute respiratory syndrome (SARS) on pulmonary function, functional capacity and quality of life in a cohort of survivors. Thorax. 2005;60(5):401-409.

2. Murthy S, Gomersall CD, Fowler RA. Care for Critically III Patients With COVID-19 [published online ahead of print, 2020 Mar 11]. Jama. 2020.

3. Yang X, Yu Y, Xu J, et al. Clinical course and outcomes of critically ill patients with SARS-CoV-2 pneumonia in Wuhan, China: a singlecentered, retrospective, observational study. The Lancet. Respiratory medicine. 2020;8(5):475-481.

4. Hui DS, Wong KT, Ko FW, et al. The 1-year impact of severe acute respiratory syndrome on pulmonary function, exercise capacity, and quality of life in a cohort of survivors. Chest. 2005;128(4):2247-2261.

5. Ngai JC, Ko FW, Ng SS, To KW, Tong M, Hui DS. The long-term impact of severe acute respiratory syndrome on pulmonary function, exercise capacity and health status. Respirology (Carlton, Vic.). 2010;15(3):543-550.

6. Herridge MS, Cheung AM, Tansey CM, et al. One-year outcomes in survivors of the acute respiratory distress syndrome. The New England journal of medicine. 2003;348(8):683-693.

7. Puthucheary ZA, Rawal J, McPhail M, et al. Acute skeletal muscle wasting in critical illness. Jama. 2013;310(15):1591-1600.

8. Hashem MD, Parker AM, Needham DM. Early Mobilization and Rehabilitation of Patients Who Are Critically III. Chest. 2016;150(3):722731.

9. Schweickert WD, Pohlman MC, Pohlman AS, et al. Early physical and occupational therapy in mechanically ventilated, critically ill patients: a randomised controlled trial. Lancet (London, England). 2009;373(9678):1874-1882.

10. National Health Commission of the People's Republic of China. Chinese management guideline for COVID-19 (version 6.0). Feb 19, 2020.

http://www.nhc.gov.cn/yzygj/s7653p/202002/8334a8326dd94d329df351d7da8aefc2/files/b218cfeb1bc54639af227f922bf6b817.pdf Accessed Feb 19, 2020; in Chinese. 
11. World Health Organization. Report of the WHO-China Joint Mission on Coronavirus Disease 2019. https://www.who.int/docs/defaultsource/coronaviruse/who-china-joint-mission-on-covid-19-final-report. Accessed Mar 5th, 2020.

12. Ranieri VM, Rubenfeld GD, Thompson BT, et al. Acute respiratory distress syndrome: the Berlin Definition. Jama. 2012;307(23):25262533.

13. Shankar-Hari M, Phillips GS, Levy ML, et al. Developing a New Definition and Assessing New Clinical Criteria for Septic Shock: For the Third International Consensus Definitions for Sepsis and Septic Shock (Sepsis-3). Jama. 2016;315(8):775-787.

14. Khwaja A. KDIGO clinical practice guidelines for acute kidney injury. Nephron. Clinical practice. 2012;120(4):c179-184.

15. Moisey LL, Mourtzakis M, Cotton BA, et al. Skeletal muscle predicts ventilator-free days, ICU-free days, and mortality in elderly ICU patients. Critical care (London, England). 2013;17(5):R206.

16. Weijs PJ, Looijaard WG, Dekker IM, et al. Low skeletal muscle area is a risk factor for mortality in mechanically ventilated critically ill patients. Critical care (London, England). 2014;18(2):R12.

17. Needham DM. Mobilizing patients in the intensive care unit: improving neuromuscular weakness and physical function. Jama. 2008;300(14):1685-1690.

18. Needham DM, Korupolu R, Zanni JM, et al. Early physical medicine and rehabilitation for patients with acute respiratory failure: a quality improvement project. Archives of physical medicine and rehabilitation. 2010;91(4):536-542.

19. Pohlman MC, Schweickert WD, Pohlman AS, et al. Feasibility of physical and occupational therapy beginning from initiation of mechanical ventilation. Critical care medicine. 2010;38(11):2089-2094.

20. Zhu C, Wu Y, Liu H, Ban Y, Ma X, Zhang Z. Early pulmonary rehabilitation for SARS-CoV-2 pneumonia: Experience from an intensive care unit outside of the Hubei province in China. Heart \& lung: the journal of critical care. 2020.

21. Liu K, Zhang W, Yang Y, Zhang J, Li Y, Chen Y. Respiratory rehabilitation in elderly patients with COVID-19: A randomized controlled study. Complementary therapies in clinical practice. 2020;39:101166.

22. Tipping CJ, Harrold M, Holland A, Romero L, Nisbet T, Hodgson CL. The effects of active mobilisation and rehabilitation in ICU on mortality and function: a systematic review. Intensive care medicine. 2017;43(2):171-183.

23. Mo X, Jian W, Su Z, et al. Abnormal pulmonary function in COVID-19 patients at time of hospital discharge. The European respiratory journal. 2020;55(6).

24. Xu Z, Shi L, Wang Y, et al. Pathological findings of COVID-19 associated with acute respiratory distress syndrome. The Lancet. Respiratory medicine. 2020;8(4):420-422.

25. Kortebein P, Ferrando A, Lombeida J, Wolfe R, Evans WJ. Effect of 10 days of bed rest on skeletal muscle in healthy older adults. Jama. 2007;297(16):1772-1774.

26. Tesch PA, von Walden F, Gustafsson T, Linnehan RM, Trappe TA. Skeletal muscle proteolysis in response to short-term unloading in humans. Journal of applied physiology (Bethesda, Md. : 1985). 2008;105(3):902-906.

27. Castro-Avila AC, Serón P, Fan E, Gaete M, Mickan S. Effect of Early Rehabilitation during Intensive Care Unit Stay on Functional Status: Systematic Review and Meta-Analysis. PloS one. 2015;10(7):e0130722.

28. Das DK, Graham ZA, Cardozo CP. Myokines in skeletal muscle physiology and metabolism: Recent advances and future perspectives. Acta physiologica (Oxford, England). 2020;228(2):e13367.

29. Huh JY. The role of exercise-induced myokines in regulating metabolism. Archives of pharmacal research. 2018;41(1):14-29.

\section{Figures}



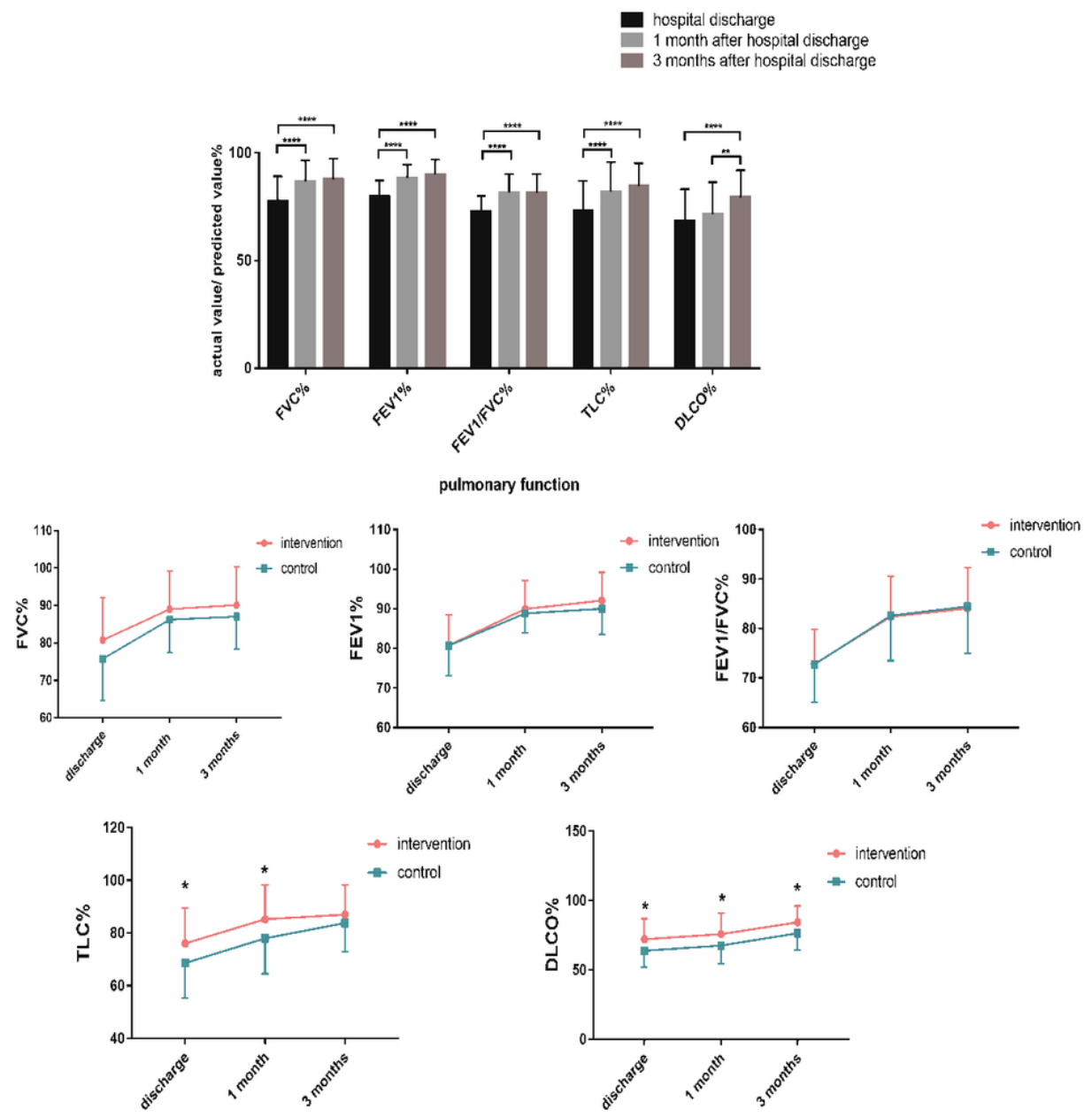

Figure 1

Pulmonary function differences between the intervention and control groups during the three-month follow-up after hospital discharge. Note. FVC\%, FEV1\%, TLC\% and DLCO\% are the percentages of observed FVC, FEV1, TLC and DLCO related to their respective predicted values. Data are presented as the mean \pm S.D: $*: 0.01 \leq p<0.05 ; * *: 0.001 \leq p<0.01 ; * \star *: 0.0001 \leq p<0.001 ;$ and $* * * *: p<0.0001$. 

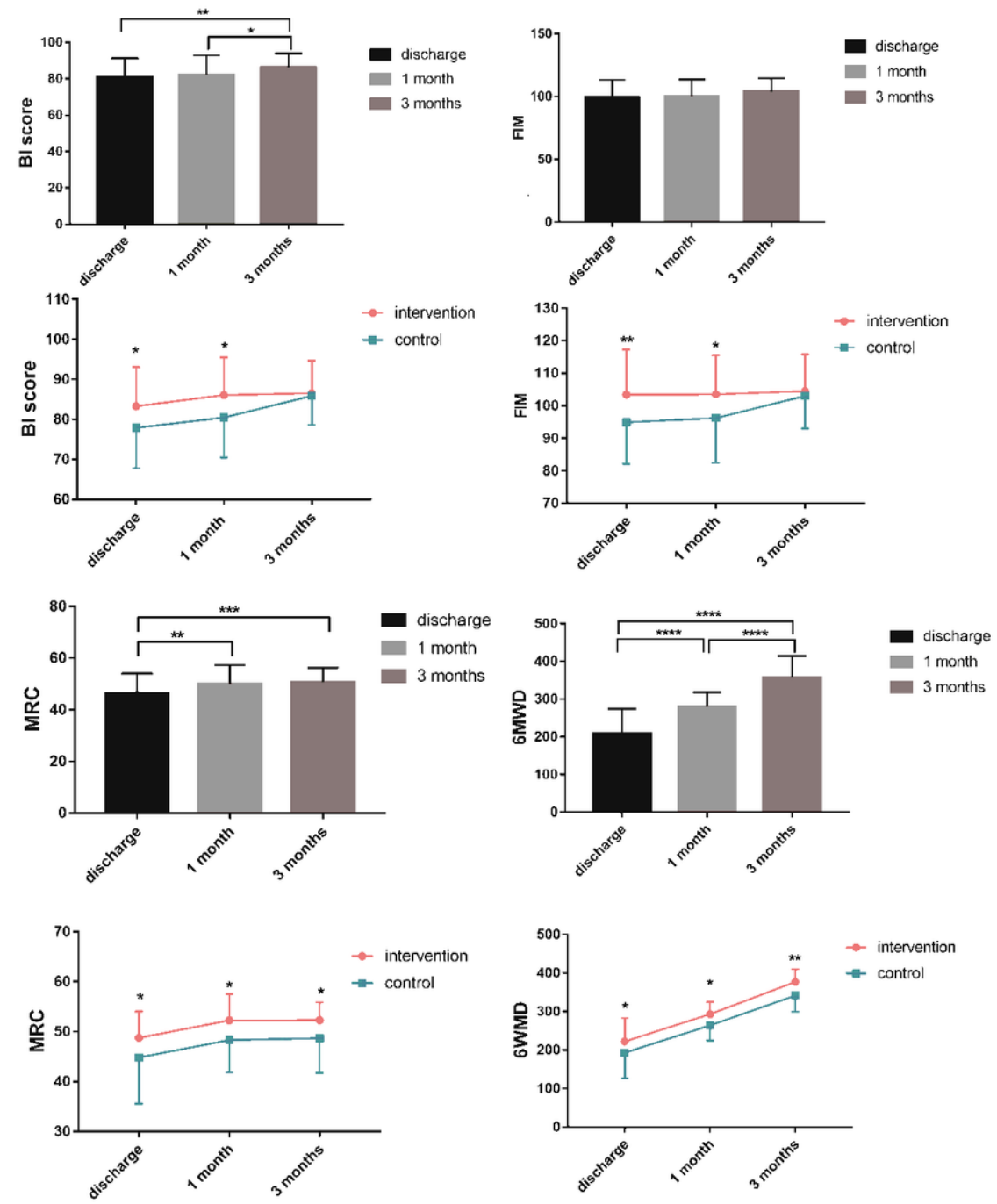

\section{Figure 2}

Differences in independent functional status, muscle strength, and exercise capacity between the intervention and control groups during the three-month follow-up after hospital discharge. Note. Data are presented as the mean \pm S.D: *: $0.01 \leq p<0.05 ; \star \star: 0.001 \leq p<0.01 ; \star \star \star$ : $0.0001 \leq p<0.001 ;$ and $* \star * *: p<0.0001$.

\section{Supplementary Files}

This is a list of supplementary files associated with this preprint. Click to download.

- supplementaryTable12.docx 